\title{
Nível de estresse e avaliação preliminar da síndrome de Burnout em Enfermeiro da UTI na COVID-19 - Estudo de caso
}

\author{
Stress level and preliminary assessment of Burnout syndrome in ICU nurses at COVID-19 - Case \\ study research
}

Nivel de estrés y evaluación preliminar del síndrome de Burnout en enfermeras de UCI en COVID19.- Estudio de caso

Ludmila Barbosa da Silva Ferreira ORCID: https://orcid.org/0000-0003-4985-654X Faculdade de Medicina de São José do Rio Preto, Brasil E-mail: lud.milabsf@gmail.com

Rita de Cassia Helú Mendonça Ribeiro

ORCID: https://orcid.org/0000-0002-1016-0484 Faculdade de Medicina de São José do Rio Preto, Brasil E-mail: ritadecassia@famerp.br Daniele Alcalá Pompeo

ORCID: https://orcid.org/0000-0003-2671-2586 Faculdade de Medicina de São José do Rio Preto, Brasil E-mail: daniele.pompeo@famerp.br

Ligia Marcia Contrin

ORCID: https://orcid.org/0000-0003-1897-2097 Faculdade de Medicina de São José do Rio Preto, Brasil E-mail: ligiacontrin@famerp.br

Alexandre Lins Werneck

ORCID: https://orcid.org/0000-0002-2911-8091 Faculdade de Medicina de São José do Rio Preto, Brasil E-mail: alexandre.werneck@ famerp.br

Renato Mendonça Ribeiro

ORCID: https://orcid.org/0000-0002-0585-7367 Faculdade de Medicina de São José do Rio Preto, Brasil E-mail: renato.mendonca.ribeiro@usp.br

Clemente Neves Sousa ORCID: https://orcid.org/0000-0003-2654-0497 Escola Superior de Enfermagem do Porto, Portugal E-mail: clementesousa@esenf.pt

\begin{abstract}
Resumo
Objetivo: Analisar o nível de estresse e sinais preliminares da Síndrome de Burnout nos enfermeiros que trabalham nas Unidades de Terapia Intensivas (UTIs) da COVID-19 e, nas demais UTIs, no contexto da pandemia. Metodologia: Estudo transversal, descritivo com abordagem quantitativo do tipo analítico, de um contexto específico, executado de fevereiro a maio de 2021, com enfermeiros das UTIs, de um Hospital de Ensino, do interior do estado de São Paulo. Foi aplicado um formulário estruturado por meio do Google Forms, associados aos Questionário de Stress nos Profissionais de Saúde de Gomes \& Teixeira e Questionário JBEILI, inspirado no Maslach Burnout Inventory - MBI (Chafic Jbeili). Para as análises estatísticas, foi adotado o nível de significância $p \leq 0,050$, sendo realizadas análises de correlação, teste de normalidade, testes estatísticos Wilcoxon, U de Mann-Whitney e Correlação de Spearman. Resultados: A população do estudo foi composta por 61 enfermeiros. Destes, 38 atuam nas UTIs COVID-19 e 23, nas demais UTIs. Não houve diferença estatística significativa, em relação ao nível de estresse e a análise preliminar de Burnout entre os dois grupos. Quanto ao nível de estresse global identificados no QSPS, a classificação de "bastante estresse" foi predominante (34\%), seguido de elevado estresse (24\%). Na análise preliminar de Burnout, 82\% da população total apresentou a Síndrome de Burnout, sendo a classificação "Fase inicial da Burnout" a mais pronunciada. Conclusão: Os resultados pontuam níveis de estresse global de "bastante" a "elevado" e predomínio da fase inicial e de instalação da síndrome de Burnout em enfermeiros das UTIs COVID-19 e demais UTIs.

Palavras-chave: Estresse emocional; Esgotamento profissional; Enfermagem; COVID-19; SARS-CoV; Unidades de Terapia Intensiva.
\end{abstract}




\begin{abstract}
Objective: Analyze the level of stress and preliminary signs of Burnout Syndrome in nurses working in Intensive Care Units (ICUs) of COVID-19 and, in other ICUs, in the context of the pandemic. Methodology: Cross-sectional, descriptive study with a quantitative analytical approach, carried out from February to May 2021, with nurses from the ICUs of a Teaching Hospital in the interior of the state of São Paulo. We applied a structured form through Google Forms, associated with the Health Professional Stress Questionnaire from Gomes \& Teixeira and the JBEILI Questionnaire, inspired by the Maslach Burnout Inventory - MBI (Chafic Jbeili). For statistical analyses, the significance level of $\mathrm{p} \leq 0.050$ was adopted, and correlation analysis, normality test, Wilcoxon statistical tests, MannWhitney U, and Spearman correlation tests were performed. Results: The study population consisted of 61 nurses. Of these, 38 work in the COVID-19 ICUs and 23 in the other ICUs. There were no statistically significant differences regarding the level of stress and the preliminary analysis of Burnout between the two groups. As for the level of global stress identified in the QSPS, the classification of high stress (34\%) predominated, followed by high stress (24\%) and, concerning the preliminary analysis of Burnout, 82\% of the total population presented the Burnout Syndrome, predominating the classification "Initial Stage of Burnout." Conclusion: The results score global stress levels from "quite" to "high" and predominance of the initial and installation phase of Burnout syndrome in nurses from COVID-19 ICUs and other ICUs. The results point to global stress levels ranging from "quite" to "high" stress, with Burnout predominating in nurses in the COVID-19 ICUs and other ICUs in the initial phase and beginning of the syndrome's onset.
\end{abstract}

Keywords: Emotional stress; Professional exhaustion; Nursing; COVID-19; SARS-CoV; Intensive Care Units.

\title{
Resumen
}

Objetivo: Analizar el nivel de estrés y signos preliminares del Síndrome de Burnout en enfermeros que laboran en las Unidades de Cuidados Intensivos (UCI) del COVID-19 y, en otras UCI, en el contexto de la pandemia. Metodología: Estudio descriptivo transversal con abordaje analítico cuantitativo, realizado de febrero a mayo de 2021, con enfermeros de las UCI de un Hospital Docente del interior del estado de São Paulo. Se aplicó un formulario estructurado através de Google Forms, asociado al Health Professional Stress Questionnaire de Gomes \& Teixeira y al JBEILI Questionnaire, inspirado en el Maslach Burnout Inventory - MBI (Chafic Jbeili). Para los análisis estadísticos se adoptó el nivel de significancia de $\mathrm{p} \leq 0,050$ y se realizaron análisis de correlación, prueba de normalidad, pruebas estadísticas de Wilcoxon, U de Mann-Whitney y pruebas de correlación de Spearman. Resultados: La población de estudio estuvo conformada por 61 enfermeras. De estos, 38 trabajan en las UCI COVID-19 y 23 en las otras UCI. No hubo diferencias estadísticamente significativas en cuanto al nivel de estrés y el análisis preliminar de Burnout entre los dos grupos. En cuanto al nivel de estrés global identificado en el QSPS, predominó la clasificación de estrés alto (34\%), seguido de estrés alto $(24 \%)$ y, en cuanto al análisis preliminar de Burnout, el $82 \%$ de la población total presentó el Síndrome de Burnout, predominando la clasificación "Etapa inicial de Burnout". Conclusión: Los resultados puntúan los niveles de estrés global de "bastante" a "alto" y predominio de la fase inicial y de instalación del síndrome de Burnout en enfermeros de UCI COVID-19 y otras UTI. Conclusion: Los resultados apuntan a niveles de estrés global que van desde "bastante" a "alto" estrés, con predominio del Burnout en enfermeras de las UCI COVID-19 y otras UCI en la fase inicial y comienzo del inicio del síndrome.

Palabras clave: Estrés emocional; Agotamiento profesional; Enfermería; COVID-19; SARS-CoV; Unidades de Cuidados Intensivos.

\section{Introdução}

Determinado pela Organização Mundial da Saúde (OMS), juntamente com o Conselho Internacional de Enfermagem, o “Ano da Enfermagem" 2020, traria maior visibilidade e valorização pública e política à profissão, a partir da campanha Nursing Now, adotada por diversos países (World Health Organization, 2018). Porém, o que atrelou à enfermagem maior visibilidade pública, não foram os esforços atribuídos à campanha e, sim, um cenário caótico e de incertezas, vigente pela pandemia, causada pelo vírus SARS-COV-2 (Costa, 2020).

E apesar de protagonistas da luta contra a COVID-19, os profissionais de enfermagem, enfrentam uma batalha antiga e diária pela valorização e condições mais adequadas ao trabalho, como a regulamentação da carga horária de 30h semanais e piso salarial. Esse último, evidenciado pelo projeto de lei no 2564, de 2020, em tramitação no Senado Federal desde 2021, em contrapartida à atual situação laboral, com jornadas extensas, baixa remuneração, múltiplas exigências e condições de trabalho diferenciadas, que se somam ao contexto da pandemia, na qual existe a tensão do perigo iminente de contágio, a falta de recursos materiais e humanos perante a demanda, a exposição a mortes em amplas proporções e o isolamento social, 
corroboraram para o aumento à vulnerabilidade de risco de adoecimento físico e mental destes profissionais (Costa, 2020; Humerez et al., 2020; Pereira et al., 2020).

Tendo em vista que a Unidade de Terapia Intensiva (UTI), decorrente de sua complexidade funcional e estrutural, se caracteriza como o setor hospitalar que mais induz ao estresse ocupacional nos profissionais de enfermagem, em conjunto com o agravamento dos sinais psiquiátricos ocasionado pela pandemia, nos leva a refletir à respeito da carga psicológica e física enfrentada pelos enfermeiros atuantes nas UTIs e na linha de frente do combate ao vírus e de como estão enfrentando e externalizando os efeitos advindos dessa sobrecarga.

Dados da OMS indicam que profissionais de enfermagem na linha de frente apresentam elevados níveis de ansiedade, depressão e estresse, atrelados ao aumento de casos da Síndrome de Burnout (SB), levando ao absenteísmo no trabalho, o que gera um impacto direto no sistema de saúde, pois cada profissional que se ausenta, gera um desfalque, sobrecarrega a equipe e influi negativamente sobre o custo e qualidade da assistência prestada (Campos et al., 2018; Humerez et al., 2020; Luziara \& Jerônimo, 2019; Prado et al., 2020; Souza e Souza, \& Souza, 2020)

Uma das principais causas do absenteísmo no trabalho, por parte da equipe de enfermagem, o estresse é caracterizado pela sensação de tensão, sobrecarga e preocupação excessiva em relação as exigências do trabalho, repercutindo no declínio do desempenho do profissional, insatisfação referente ao trabalho, acidentes no exercer da profissão, problemas cardiovasculares, distúrbios psíquicos menores e quando crônico, evolui para a Síndrome de Burnout, sendo esta, um fenômeno ocupacional, reconhecida pela Classificação Estatística Internacional de Doenças e Problemas Relacionados com a Saúde, em sua décima primeira edição (CID-11), na qual é resultante do estresse crônico em ambiente de trabalho e caracterizada por 3 dimensões: sentimentos de exaustão ou esgotamento de energia; Distanciamento mental do próprio trabalho, ou sentimentos de negativismo e cinismo em relação ao trabalho e falta de realização e redução da eficácia profissional; (Scholze et al., 2017; Luziara \& Jerônimo, 2019; )

Pelas razões apresentadas no texto, em relação as repercussões negativas dos níveis de estresse elevados e aumento dos casos da síndrome de Burnout nos profissionais da saúde, em presente contexto, destaca-se a importância de identificar sinais de estresse e fatores que desencadeiam tal condição, bem como a identificação preliminar da Síndrome de Burnout, nos enfermeiros que atuam nas Unidades de Terapia intensiva (UTIs) no contexto da pandemia, trazendo relevância, para que medidas preventivas e de acolhimento terapêutico possam ser direcionadas. Portanto, o objetivo da presente pesquisa, foi analisar o nível de estresse e sinais preliminares da Síndrome de Burnout nos enfermeiros que trabalham nas Unidades de Terapia Intensivas (UTIs) da COVID-19 e, nas demais UTIs, no contexto da pandemia.

\section{Metodologia}

Estudo de caso transversal com delineamento descritivo, abordagem quantitativa do tipo analítica com correlação entre as variáveis, realizado com enfermeiros dos setores de Unidade de Terapia Intensiva Adulto, em um hospital escola do interior do Estado de São Paulo, sendo os dados pontuados nesta pesquisa, específicos do contexto investigado, não podendo, portanto, serem analisados de modo generalista.

A população inicial foi composta por 111 enfermeiros, atuantes nas dez unidades de terapia intensiva, sendo encaminhado o formulário a estes. Dos 111 enfermeiros, 68 atuavam nas UTIs voltadas para o cuidado dos pacientes com COVID-19 e, 43, atuavam nas demais UTIs. No período da coleta de dados, 12 enfermeiros estavam afastados, assim a população do estudo final foi composta por 99 enfermeiros. Houve uma alteração, tanto no quantitativo de enfermeiros que atuam nas UTIs, quanto ao quantitativo de leitos em relação à antes do contexto da pandemia, passando de 62 para 122 enfermeiros e 117 leitos para 225 leitos durante o pico da pandemia. 
Dos 99 enfermeiros, 61 responderam o Google Forms ${ }^{\circledR}$. A coleta de dados foi realizada no período de fevereiro a maio de 2021. Os questionários foram aplicados via formulário eletrônico e enviados no e-mail do profissional. Os dados referentes aos questionários foram computados e analisados pela própria plataforma, com uso de uma planilha específica. Foram aplicados três questionários: um referente aos dados sociodemográficos e contexto laboral, para descrição do perfil da população estudada, um segundo para avaliar o nível de estresse e, um terceiro, para a identificação preliminar da Síndrome de Burnout.

Os critérios de inclusão foram todos os enfermeiros que atuavam nas unidades de terapia intensiva (UTIs) sendo critérios de exclusão, enfermeiros de férias e aqueles que estavam em licença no período da coleta de dados.

Para coleta de dados sociodemográficos e contexto atual profissional, como, qual a unidade de terapia intensiva que atual, há quanto tempo se graduou e trabalha na instituição, qual a carga horária de trabalho, qual o nível de insegurança perante o contexto da pandemia, se sentem apoiados pela instituição na qual trabalham e como classificam a remuneração na qual recebem, frente ao trabalho exercido, sendo as classificações: "insatisfatório", "regular" e "satisfatório." A segunda parte estava voltada para os profissionais atuantes nas UTIs COVID-19, na qual correspondiam a perguntas referentes a experiência e atuação em unidade de terapia intensiva, anterior ao contexto da pandemia, se foi realocado de setor, se sente apto a atuar em uma UTI voltada para os cuidados de pacientes com a COVID-19, qual o nível de insegurança frente ao risco de contaminação ao contato direto, se sentem desconfortos ao atuarem no setor, se sim, quais e se sentem necessidade de intervenções para mediar o contexto da COVID-19.

Para analisar o nível de estresse, aplicamos o questionário "Health Professional Stress Questionnaire" (Gomes, \& Teixeira, 2016). Usamos o Questionário de Jbeili para identificação preliminar da Síndrome de Burnout. O questionário foi elaborado e adaptado por Chafic Jbeili, inspirado no Maslach Burnout Inventory - MBI (Jbeili, 2011). Todos os instrumentos de coleta, foram adaptados ao Google Forms ${ }^{\circledR}$.

O Questionário de estresse, aplicado aos profissionais de saúde (QSPS), foi desenvolvido por Gomes e Teixeira (2016) com base nos trabalhos originais de Gomes (1998) e Gomes, Cruz e Cabanelas (2009) aplicados em domínios diferentes de profissionais da área da saúde (psicólogos, médicos, enfermeiros, entre outros), obtendo boas indicações sobre as propriedades psicométricas. (Gomes \& Teixeira, 2016; Machado,2020)

O QSPS compreende duas partes distintas, sendo a primeira, correlacionada à avaliação do nível global de estresse, por meio de um único item (Zero = Nenhum estresse; Um= pouco estresse; Dois = Moderado estresse; Três= bastante estresse; Quatro = Elevado estresse $s$ ). Na segunda seçção, foram relacionados 25 itens referentes a potenciais fontes de estresse, vinculados à atividade profissional, sendo os itens distribuídos em seis subescalas (Um- Lidar com clientes, Dois- Excesso de trabalho, Três- Carreira e remuneração, Quatro- Relações profissionais, Cinco- Ações de formação e Seis- Problemas familiares) as respostas são colocadas em uma escala tipo Likert de cinco pontos (Zero = Nenhum stress; $\mathrm{Um}=$ pouco stress; Dois $=$ Moderado stress $;$ Três $=$ bastante stress $;$ Quatro $=$ Elevado stress $)$.

A pontuação é obtida por meio da soma dos itens de cada dimensão, dividindo-se depois os valores pelo total de itens da subescala respectiva, sendo os valores mais elevados com significância de maior percepção de estresse em relação aos domínios avaliados.

Os resultados encontrados em relação ao QPSP, foram pontuados entre os dois grupos desta pesquisa, enfermeiros (as) que atuam nas UTIs COVID-19 e enfermeiros (as) que atuam nas demais UTIs.

Os resultados encontrados em relação ao QPSP, foram pontuados entre os dois grupos desta pesquisa, enfermeiros (as) que atuam nas UTIs COVID-19 e enfermeiros (as) que atuam nas demais UTIs.

O questionário Jbeili- adaptado por Chafic Jbeili, inspirado no Maslach Burnout Inventory - MBI, está estruturado com 20 itens, respondidos por meio de uma escala de pontos, de zero a cinco, correspondendo: Um- Nunca; Dois- 
Anualmente; Três- Mensalmente; Quatro-Semanalmente e cinco-Diariamente. Ao final, soma-se a quantificação dada pela frequência assinalada em cada um dos 20 itens, dispostas nos scores a seguir: De 0 a 20 pontos: nenhum indício da Burnout; de 21 a 40 pontos: possibilidade de desenvolver Burnout; de 41 a 60 pontos: fase inicial da Burnout; de 61 a 80 pontos: a Burnout começa a se instalar; e de 81 a 100 pontos: fase considerável da Burnout.

Após a tabulação dos dados foi realizada a análise estatística, na qual foram exercidas duas funções de análises estatísticas: Descritiva e Inferencial.

$\mathrm{Na}$ análise descritiva foi traçado o perfil da população estudada, contemplando as variáveis analisadas e seus desdobramentos. No âmbito inferencial, foi traçado como objetivo estatístico, a análise de independência e predição entre as variáveis propostas, utilizando, dentro dos padrões esperados, os testes Wilcoxon, U de Mann-Whitney e Correlação de Spearman.

Os resultados de independência entre as variáveis propostas, se deram por meio da análise entre os valores de $\mathrm{P}$ (significância), em que $<0,05$, caracterizou significância entre os grupos estudados. Os testes contemplam erro alfa de $5 \%$ e confiabilidade de $95 \%$. Todos as análises foram obtidas por meio do Software SPSS Statistics (versão 23) atreladas as funcionalidades da ferramenta Excel (versão 2016).

A pesquisa foi aprovada pelo Comitê de Ética em Pesquisa da Faculdade de Medicina de São José do Rio Preto (CEP/FAMERP), Parecer $\mathrm{n}^{\circ} 4.397 .923$, e o financiamento se deu por recursos próprios dos autores. Os participantes leram e assinaram (Consentiram) o Termo de Consentimento livre e esclarecido (TCLE), disponibilizado no Google Forms ${ }^{\circledR}$, seguindo a portaria 466/12 do Ministério da Saúde do Brasil.

\section{Resultados}

No questionário dos dados sociodemográficos e contexto ambiental e profissional, dos formulários encaminhados, 61 foram preenchidos. Destes, 38 (62\%) foram respondidos pelos enfermeiros que atuavam na UTI Covid 19 e, 23 (38\%), pelos enfermeiros que atuavam nas demais UTIs (sendo sete na UTI geral, cinco na UTI convênio, cinco na UTI cardiológica, três no Pós-Operatório Imediato, dois na UTI neuro e um na UTI semi-intensiva). Em relação aos dados sociodemográficos, a população é majoritariamente do sexo feminino, na faixa etária dos 25 a 35 anos (43/70,5\%); os demais estão entre a faixa etária de 20 a 25 anos $(6 / 9,8 \%)$ e de 35 anos ou mais (12/19,6\%).

Quanto ao estado civil, predomina o casado $(33 / 54,1 \%)$, seguido do solteiro $(20 / 32,8 \%)$, união estável $(5 / 8,2 \%)$ e divorciado (3/4,9\%). A maioria da população reside em São José do Rio Preto (39/64\%) e, 22/36\%, residem na região. Dos residentes na região, 13 (59\%) são enfermeiros atuantes na linha de frente contra a COVID-19. Da população total, 21 (34\%) residem com alguém considerado do grupo de risco. Destes 21, 13 (62\%) são enfermeiros que atuam na linha de frente contra a COVID-19.

Em relação ao tempo de formação, a média dos enfermeiros é de 7,97 \pm 5,08 anos de formação, sendo o máximo de 23 anos de formação e mínimo relacionado a menos de um ano de formado. Quanto aos enfermeiros atuantes na linha de frente, foi perceptível uma variação de menos de um ano de formado até o máximo, 16 anos, de formados. Quanto aos que atuam nas demais UTIs, o tempo de formado varia de um ano até 23 anos.

Para a variável "Há quantos anos trabalha na instituição?", a média dos enfermeiros é de 4,62 \pm 4,80 anos, sendo o mínimo de menos de um ano e o máximo de 20 anos trabalhados na instituição. Dos 38 enfermeiros que atuavam na linha de frente, é perceptível que há novos enfermeiros atuantes na instituição com menos de um ano de trabalho (cinco/13\%). Os enfermeiros com mais anos de trabalho tinham 15 anos de instituição. Os 23 enfermeiros que atuavam em outras UTIs, tinham tempo de atuação variando de 1 a 20 anos. 
Nota-se uma carga horária diária predominante de seis horas, tanto para os enfermeiros que atuavam nas UTIs COVID-19 (22/ 58\%) quanto para aqueles que atuavam nas demais UTIs (15/ 65\%) com média de 7,90 $\pm 2,64$ horas. Em relação à linha de frente, a segunda carga horária diária de trabalho mais presente é a de 12 horas, (escala de 12 horas de trabalho, seguida de 36 horas de descanso) (14/37\%). Nas demais UTIs, predomina a escala de oito horas por dia $(5 / 22 \%)$. Ressalta-se que durante a pandemia, houve o rearranjo da carga horária de trabalho por meio de horas extras trabalhadas.

O Gráfico 1, abaixo, demonstra a classificação do total de enfermeiros (61), em escala por nota em relação ao item "Em uma escala de zero a dez, o quão inseguro se sente, quanto ao risco de contaminação pelo SARS-COV-2", sendo notório o nível de insegurança pontuado entre as notas maiores, relacionadas ao grau de insegurança com média de 6,34 $\pm 2,80$.

\section{Gráfico 1.}
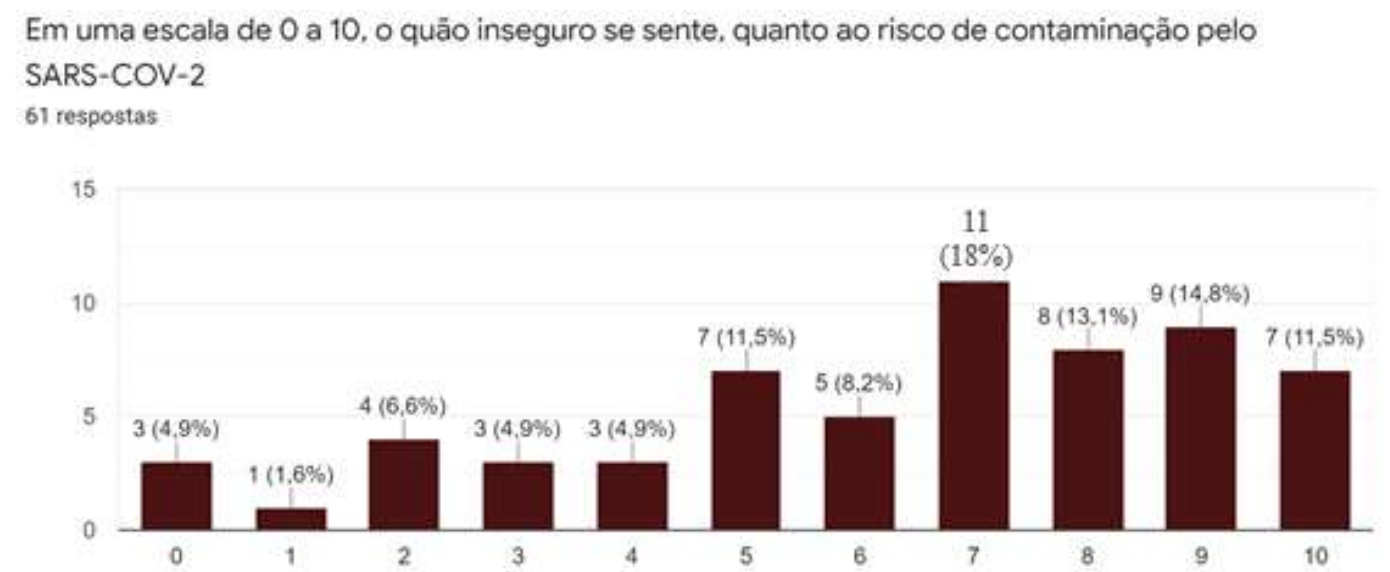

Relacionado a pontuação atribuída ao item: Em uma escala de 0 a 10, o quão inseguro se sente, quanto ao risco de contaminação pelo SARSCOV-2, referente a enfermeiros que atuam nas UTIs COVID-19 e demais UTIs de um hospital no interior São Paulo. 2021. Fonte: Autores.

Referente à classificação quanto a remuneração frente ao trabalho exercido, pela amostra total, segue predominante a classificação regular (31/51\%), seguido de satisfatório (18/ 29\%) e insatisfatório (12/20\%).

A maioria (57/93\%) sente-se apoiada pela instituição na qual trabalha, quatro (7\%) não se sentem apoiados e destes, dois atuam na linha de frente. Os quatro apresentaram notas de insegurança quanto ao risco de contaminação variando de cinco a oito. A classificação quanto à remuneração, variou de regular a insatisfatório.

Dos 38 enfermeiros que atuavam nas UTIs COVID-19, todos se sentem aptos a atuarem na área. Destes, 27 (71\%) estão atuando em sua área de especialização (UTI) e 11 (29\%) declaram não estarem atuando em seu ambiente de especialização; oito não tinham experiências anteriores no setor da UTI e, antes de estarem atuando na UTI- COVID-19, quatro atuavam no centro cirúrgico, um na emergência, um na emergência/auditoria, dois nas enfermarias, um na hemodiálise, um no centro cirúrgico e pronto-socorro e um no setor de exames.

Quanto ao item "Em uma escala de zero a dez, o quão inseguro se sente, quanto a exposição direta e o risco de contaminação pelo SARS-COV-2" predominou a nota 9 (n=8) com média 6,34 $\pm 2,93$, o restante variou conforme o Gráfico 2. 


\section{Gráfico 2.}

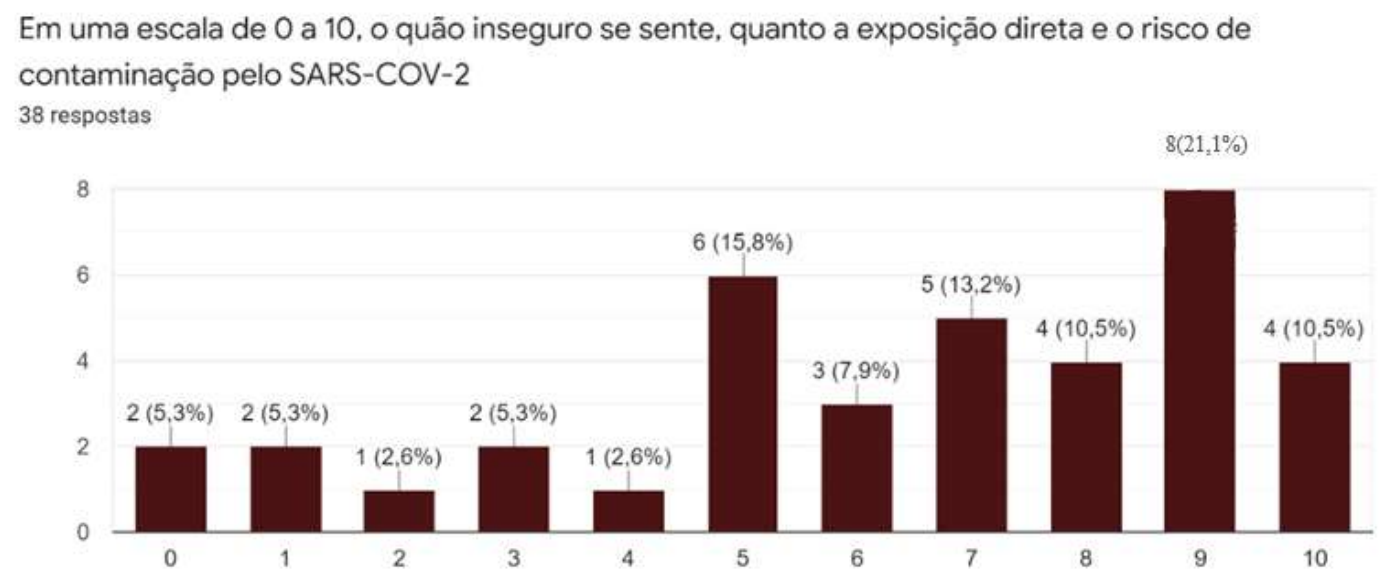

Relacionado a pontuação atribuída ao item: Em uma escala de 0 a 10, o quão inseguro se sente, quanto a exposição direta e o risco de contaminação pelo SARS-COV-2, referente a enfermeiros que atuam nas UTIs COVID-19 de um hospital no interior São Paulo. 2021. Fonte: Autores.

Em relação ao desconforto, seja emocional ou físico, ao trabalhar na linha de frente no combate ao Coronavírus, 50\% (19) dos enfermeiros responderam que sentem algum desconforto e citaram o estresse, o medo de se contaminar e contaminar pessoas próximas, com uma frequência de $21 \%$ em relação as respostas. O cansaço físico, preocupação, sobrecarga de trabalho, ansiedade, tristeza e impotência teve uma frequência de 10,5\% e exaustão, desânimo, desconforto emocional, desconforto referente a paramentação e as mortes, $5,2 \%$ das respostas. Ao serem questionados sobre sentirem a necessidade de intervenções para mediarem a situação vigente, dos 38 enfermeiros que responderam, 68,4\% (24) relataram que sentem necessidade de intervenções.

Quanto a aplicação do Questionário de Stress aos profissionais de saúde (Gomes, \& Teixeira, 2016), nos resultados da primeira seção de perguntas, referente ao nível de estresse global, predominou a classificação de bastante estresse (34\%/21), seguido de elevado estresse (24\%/ 17) dentro da amostra em geral de enfermeiros (61). Sendo a classificação dos níveis de estresse global nos grupos, da seguinte forma, dos 38 enfermeiros que atuam nas UTIs COVID-19, 39\% (15) apontaram a classificação de "bastante estresse", 26\% (dez) "moderado estresse". 18\% (sete) "elevado estresse", 13\%(cinco) "pouco estresse" e 3\% (um) "nenhum estresse 3\% (um). Já em relação aos enfermeiros que atuavam nas demais UTIs (n=23), 43\% (dez) apontaram a classificação de elevado estresse, seguida de bastante estresse $26 \%$ (seis), moderado estresse 22\% (cinco) e pouco estresse $9 \%$ (dois).

Os resultados referentes a segunda secção do QSPS, relacionado as subescalas, conforme cada grupo de profissionais (os que atuam nas UTIs COVID-19 e os que atuam nas demais UTIs) estão descritos na tabela 1. 
Tabela 1: Score subescalas secção 2 do Questionário de stress nos profissionais de saúde, relacionado a enfermeiros das UTIs COVID-19 e demais UTIs, de um hospital localizado no interior de SP. 2021

\begin{tabular}{|c|c|c|c|c|c|c|c|c|c|c|c|c|}
\hline \multicolumn{13}{|c|}{ UTI COVID-19 } \\
\hline \multirow[b]{3}{*}{ Escala } & \multirow{3}{*}{$\begin{array}{c}1 . \\
\text { Lidar } \\
\text { com } \\
\text { Clien- } \\
\text { tes }\end{array}$} & \multirow[b]{3}{*}{$\%$} & \multirow[b]{3}{*}{$\begin{array}{c}2 . \\
\text { Excesso de } \\
\text { trabalho }\end{array}$} & \multirow[b]{3}{*}{$\%$} & \multirow{3}{*}{$\begin{array}{c}3 . \\
\text { Carreira } \\
\text { e } \\
\text { remune- } \\
\text { ração }\end{array}$} & \multirow[b]{3}{*}{$\%$} & \multirow{3}{*}{$\begin{array}{c}4 . \\
\text { Relações } \\
\text { profissio- } \\
\text { nais }\end{array}$} & \multirow[b]{3}{*}{$\%$} & \multirow[b]{3}{*}{$\begin{array}{c}5 . \\
\text { Ações de } \\
\text { formação }\end{array}$} & \multirow{3}{*}{\multicolumn{2}{|c|}{$\begin{array}{c}6 . \\
\text { Proble- } \\
\text { mas } \\
\text { familiares }\end{array}$}} & \multirow[b]{3}{*}{$\%$} \\
\hline & & & & & & & & & & & & \\
\hline & & & & & & & & & & & & \\
\hline 0: nenhum & & & & & & & & & & 11 & & \\
\hline estresse & 1 & $3 \%$ & 0 & $0 \%$ & 1 & $3 \%$ & 1 & $3 \%$ & 4 & $\%$ & 3 & $8 \%$ \\
\hline 1: Pouco & & & & & & & & 26 & & 45 & & \\
\hline estresse & 2 & $5 \%$ & 7 & $18 \%$ & 9 & $24 \%$ & 10 & $\%$ & 17 & $\%$ & 10 & $26 \%$ \\
\hline \multicolumn{13}{|l|}{ 2: } \\
\hline Moderado & & 34 & & & & & & 42 & & 32 & & \\
\hline estresse & 13 & $\%$ & 11 & $29 \%$ & 12 & $32 \%$ & 16 & $\%$ & 12 & $\%$ & 16 & $42 \%$ \\
\hline 3: Bastante & & 34 & & & & & & 26 & & 13 & & \\
\hline estresse & 13 & $\%$ & 13 & $34 \%$ & 13 & $34 \%$ & 10 & $\%$ & 5 & $\%$ & 6 & $16 \%$ \\
\hline 4: Elevado & & 24 & & & & & & & & & & \\
\hline estresse & 9 & $\%$ & 7 & $18 \%$ & 3 & $8 \%$ & 1 & $3 \%$ & 0 & $0 \%$ & 3 & $8 \%$ \\
\hline \multirow[b]{2}{*}{ Total } & & 100 & & & & & & 100 & & 100 & & \\
\hline & 38 & $\%$ & 38 & $100 \%$ & 38 & $100 \%$ & 38 & $\%$ & 38 & $\%$ & 38 & $100 \%$ \\
\hline \multicolumn{13}{|c|}{ Demais UTIs } \\
\hline & 1. & & & & 3. & & & & & & & \\
\hline & Lidar & & & & Carreira & & 4. & & & & & \\
\hline & com & & 2. & & e & & Relações & & 5. & & 6. Proble- & \\
\hline Escala & $\begin{array}{l}\text { Clien- } \\
\text { tes }\end{array}$ & $\%$ & $\begin{array}{c}\text { Excesso de } \\
\text { trabalho }\end{array}$ & $\%$ & $\begin{array}{l}\text { remune- } \\
\text { ração }\end{array}$ & $\%$ & $\begin{array}{l}\text { profissio- } \\
\text { nais }\end{array}$ & $\%$ & $\begin{array}{l}\text { Ações de } \\
\text { formação }\end{array}$ & $\%$ & $\begin{array}{c}\text { mas } \\
\text { familiares }\end{array}$ & $\%$ \\
\hline \multicolumn{13}{|l|}{ 0: nenhum } \\
\hline estresse & 0 & $0 \%$ & 0 & $0 \%$ & 1 & $4 \%$ & 0 & $0 \%$ & 1 & $4 \%$ & 1 & $4 \%$ \\
\hline 1: Pouco & & & & & & & & & & 22 & & \\
\hline estresse & 1 & $4 \%$ & 2 & $9 \%$ & 5 & $22 \%$ & 2 & $9 \%$ & 5 & $\%$ & 7 & $30 \%$ \\
\hline \multicolumn{13}{|l|}{$2:$} \\
\hline Moderado & & 30 & & & & & & 48 & & 57 & & \\
\hline estresse & 7 & $\%$ & 3 & $13 \%$ & 7 & $30 \%$ & 11 & $\%$ & 13 & $\%$ & 4 & $17 \%$ \\
\hline 3: Bastante & & 43 & & & & & & 35 & & 17 & & \\
\hline estresse & 10 & $\%$ & 15 & $65 \%$ & 8 & $35 \%$ & 8 & $\%$ & 4 & $\%$ & 8 & $35 \%$ \\
\hline 4: Elevado & & 22 & & & & & & & & & & \\
\hline estresse & 5 & $\%$ & 3 & $13 \%$ & 2 & $9 \%$ & 2 & $9 \%$ & 0 & $0 \%$ & 3 & $13 \%$ \\
\hline & & 100 & & & & & & 100 & & 100 & & \\
\hline Total & 23 & $\%$ & 23 & $100 \%$ & 23 & $100 \%$ & 23 & $\%$ & 23 & $\%$ & 23 & $100 \%$ \\
\hline
\end{tabular}

Fonte: Autores.

Observa-se que a classificação de estresse entre as subescalas e os grupos coincidem, sendo diferentes, nas subescalas cinco (ações de formação) na qual para os profissionais que atuam na UTIs Covid 19 geram pouco estresse correspondente a $45 \%$ (17) da amostra e nos profissionais que atuam nas demais UTIs é um fator que gera moderado estresse correspondendo a $57 \%$ (13) da amostra e subescala seis (Problemas familiares) na qual para os profissionais que atuam nas UTIs COVID-19 é fator que gera moderado estresse (16/42\% da amostra) e para os que atuam nas demais UTIs é um fator com resultados bem divididos, na qual temos a classificação de bastante estresse com 35\% (oito) da amostra e fator de pouco estresse correspondendo a $30 \%$ (sete) da amostra.

Os resultados dos scores de estresse tanto relacionado a secções um do QSPS, como em cada subescalas da secção dois, entre os dois grupos, quando comparados e analisados estatisticamente, não demonstram diferenças significativas, sendo aplicado o teste de normalidade Komolgorov-Smirnov e o teste estatístico wilcoxon, com resultado de P, na secção um (Score global de estresse) de 0,066 e secção dois correspondentes as subescalas (um, dois, três, quatro, cinco e seis) foram respectivamente de 0,$520 ; 0,225 ; 0,624 ; 0,101 ; 0,174,0,505$.

No âmbito geral, quanto a amostra total (61), predominou na secção um, o score de bastante estresse (21/ 34\%), seguido de elevado estresse (17/ 28\%) e moderado estresse (15/25\%), na secção dois, referente a subescala um-lidar com 
clientes variou minimamente entre moderado a bastante estresse (respectivamente 20/33\% e 23/ 38\% da amostra total), subescala dois-Excesso de trabalho, predominou bastante estresse (28/46\%), subescala três-Carreira e remuneração variou minimamente entre moderado a bastante estresse (respectivamente $19 / 31 \%$ e $21 / 34 \%$ da amostra total),subescala quatroRelações profissionais predominou o escore moderado estresse (27/ 44\% da amostra total), subescala cinco- Ações de formação, variou minimamente entre pouco a moderado estresse (respectivamente $22 / 36 \%$ e 25/ $41 \%$ da amostra total) e subescala seis- Problemas familiares, variou minimamente entre pouco a moderado estresse (respectivamente 17/ 28\% e 20/ $33 \%$ da amostra total).

Ao executar o questionário JBEILI- adaptado por Chafic Jbeili, inspirado no Maslach Burnout Inventory - MBI, predominou o score: "Fase inicial da Burnout" nos dois grupos, sendo em $61 \%$ em relação a amostra dos profissionais que atuam em UTIs COVID-19 (38) e 65\% da amostra dos que atuam nas demais UTIs (23). Na amostra dos profissionais que atuam nas UTIs COVID-19, o segundo score mais predominante foi o de Possibilidade de desenvolver Burnout (24\%) seguido de: A Burnout começa a se instalar (16\%/6), já na amostra referente a profissionais que atuam nas demais UTIs depois do score mais predominante, pontua-se o score: A Burnout começa a se instalar (26\%/6) seguido de possibilidade de desenvolver Burnout (9\%/2). Não houve pontuação para nenhum dos extremos (nenhum indício e fase considerável da Burnout) relacionado ao score do questionário. Segue em gráfico 3, as classificações dos scores em cada grupo.

Gráfico 3. Score JBEILI em relação a amostra.

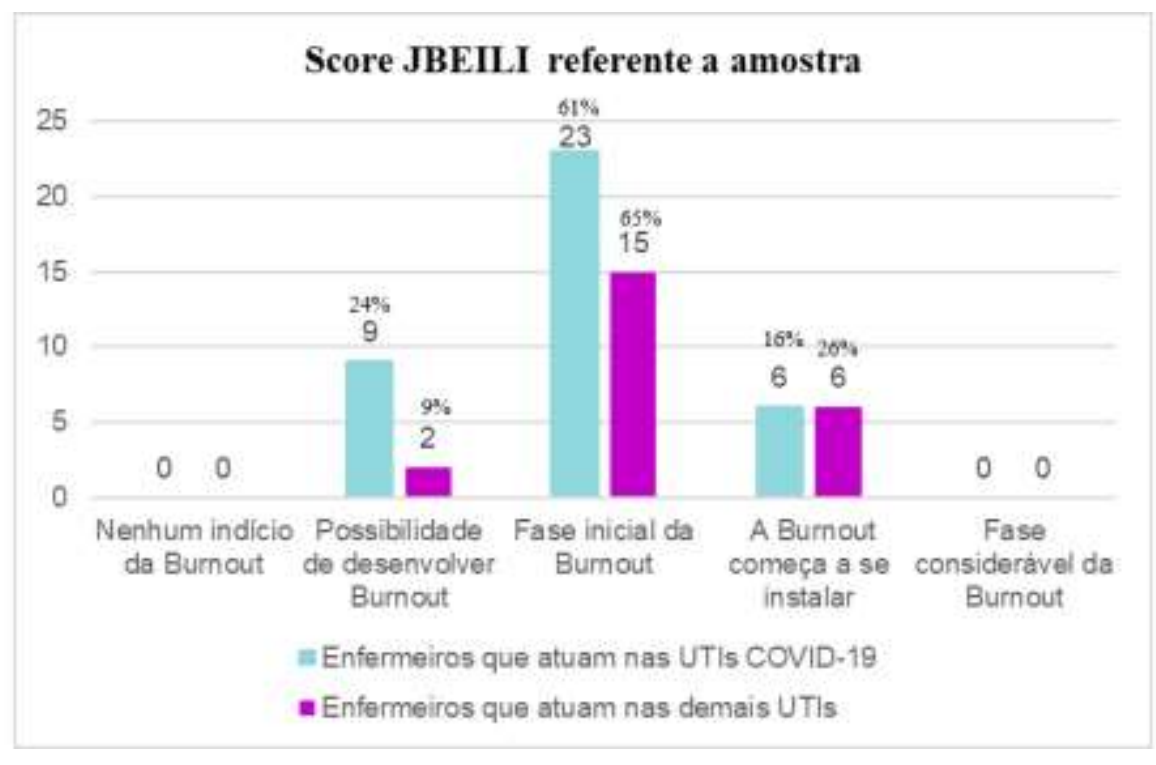

Fonte: Autores.

A comparação do resultado entre os dois grupos não apresentou diferença significativa em relação a análise estatística, sendo aplicado Teste Wilcoxon com $\mathrm{P}=0,66$. No âmbito geral, do total de enfermeiros (61), há uma prevalência da síndrome em $82 \%$ (50) do total de enfermeiros, e 18\% (11) tem potencial para desenvolver a síndrome, demonstrando a importância de medidas de intervenção para que os casos não se agravem e de forma preventiva para que os que tem a possibilidade de desenvolver.

Após a pontuação do score referente ao questionário JBEILI (análise preliminar da Síndrome de Burnout) foi realizado a análise estatística da correlação do score com dados sociodemográficos conforme Tabela 2. 
Tabela 2: Comparação de Score de Burnout com Variáveis sociodemográficas, relacionadas a enfermeiros(as) que atuam em nas UTIs de um hospital, localizado no interior de São Paulo, 2021.

\begin{tabular}{|c|c|c|c|c|c|c|c|c|c|}
\hline \multirow{4}{*}{ Informações } & \multicolumn{9}{|c|}{ Definição de Score de Burnout } \\
\hline & \multicolumn{2}{|c|}{ Total } & \multicolumn{2}{|c|}{$\begin{array}{c}\text { A Burnout começa } \\
\text { a se instalar }\end{array}$} & \multicolumn{2}{|c|}{$\begin{array}{l}\text { Fase inicial da } \\
\text { Burnout }\end{array}$} & \multicolumn{2}{|c|}{$\begin{array}{c}\text { Possibilidade de } \\
\text { desenvolver } \\
\text { Burnout }\end{array}$} & \multirow[t]{3}{*}{$\begin{array}{c}\text { Valor } \\
P\end{array}$} \\
\hline & $N$ & $\%$ & $N$ & $\%$ & $N$ & $\%$ & $N$ & $\%$ & \\
\hline & 61 & 100,00 & 12 & 19,67 & 38 & 62,30 & 11 & 18,03 & \\
\hline \multicolumn{10}{|l|}{ Idade } \\
\hline 20 a 25 anos & 6 & 9,84 & 1 & 8,33 & 5 & 13,16 & 0 & 0,00 & \multirow{5}{*}{0,383} \\
\hline 25 a 30 anos & 21 & 34,43 & 2 & 16,67 & 16 & 42,11 & 3 & 27,27 & \\
\hline 30 a 35 anos & 22 & 36,07 & 4 & 33,33 & 12 & 31,58 & 6 & 54,55 & \\
\hline 35 a 40 anos & 6 & 9,84 & 1 & 8,33 & 4 & 10,53 & 1 & 9,09 & \\
\hline$>40$ anos & 6 & 9,84 & 4 & 33,33 & 1 & 2,63 & 1 & 9,09 & \\
\hline \multicolumn{10}{|l|}{ Estado Civil } \\
\hline Casado & 33 & 54,10 & 9 & 75,00 & 19 & 50,00 & 5 & 45,45 & \multirow{4}{*}{0,880} \\
\hline Divorciado & 3 & 4,92 & 0 & 0,00 & 3 & 7,89 & 0 & 0,00 & \\
\hline Solteiro & 20 & 32,79 & 3 & 25,00 & 11 & 28,95 & 6 & 54,55 & \\
\hline União Estável & 5 & 8,20 & 0 & 0,00 & 5 & 13,16 & 0 & 0,00 & \\
\hline
\end{tabular}

Em relação a graduação, há quantos anos se formou?

\begin{tabular}{|c|c|c|c|c|c|c|c|c|c|}
\hline Até 5 anos & 21 & 34,43 & 3 & 25,00 & 15 & 39,47 & 3 & 27,27 & \multirow{4}{*}{0,127} \\
\hline 6 a 10 anos & 25 & 40,98 & 4 & 33,33 & 14 & 36,84 & 7 & 63,64 & \\
\hline 11 a 15 anos & 10 & 16,39 & 3 & 25,00 & 6 & 15,79 & 1 & 9,09 & \\
\hline$>15$ anos & 5 & 8,20 & 2 & 16,67 & 3 & 7,89 & 0 & 0,00 & \\
\hline \multicolumn{10}{|c|}{$\begin{array}{c}\text { Há quantos anos trabalha na } \\
\text { instituição? }\end{array}$} \\
\hline Até 5 anos & 41 & 67,21 & 4 & 33,33 & 29 & 76,32 & 8 & 72,73 & \multirow{4}{*}{$\mathbf{0 , 0 0 0}$} \\
\hline 6 a 10 anos & 13 & 21,31 & 4 & 33,33 & 6 & 15,79 & 3 & 27,27 & \\
\hline 11 a 15 anos & 6 & 9,84 & 3 & 25,00 & 3 & 7,89 & 0 & 0,00 & \\
\hline$>15$ anos & 1 & 1,64 & 1 & 8,33 & 0 & 0,00 & 0 & 0,00 & \\
\hline \multicolumn{10}{|c|}{$\begin{array}{c}\text { Qual a carga horária de trabalho } \\
\text { por dia? }\end{array}$} \\
\hline 6 horas & 37 & 60,66 & 9 & 75,00 & 23 & 60,53 & 5 & 45,45 & \multirow{3}{*}{0,271} \\
\hline 8 horas & 7 & 11,48 & 2 & 16,67 & 5 & 13,16 & 0 & 0,00 & \\
\hline 12 horas & 17 & 27,87 & 1 & 8,33 & 10 & 26,32 & 6 & 54,55 & \\
\hline
\end{tabular}


Como você classifica, a remuneração

que recebe, frente ao trabalho exercido

\begin{tabular}{cccccccccc}
\hline Insatisfatório & 12 & 19,67 & 4 & 33,33 & 6 & 15,79 & 2 & 18,18 \\
\hline Regular & 31 & 50,82 & 6 & 50,00 & 20 & 52,63 & 5 & 45,45 & $\mathbf{0 , 0 3 1}$ \\
\hline Satisfatório & 18 & 29,51 & 2 & 16,67 & 12 & 31,58 & 4 & 36,36 \\
\hline
\end{tabular}

Fonte: Autores.

Por meio dos testes estatísticos aplicados em cada variável, pode se constatar que não houve relevância significativa entre a correlação do score de Burnout com as seguintes variáveis: idade, estado civil, tempo de formação. Embora os dados encontrados demonstrem uma relevância clínica, sendo que a correlação de Spearman aplicada evidenciou que há tendencias dentro deste estudo. Onde a correlação do maior score de Burnout foi da faixa etária entre 25 a 35 anos, de enfermeiros com até 10 anos formação, com até cinco anos de trabalho na instituição $p=(0,000)$ e menor carga horária de trabalho (escala diária de 6h), com maior predominância na fase inicial da Síndrome de Burnout.

Das variáveis correlacionadas, apenas a variável correspondente a classificação da remuneração frente ao trabalho exercido, apresentou significância com a correlação do score de Burnout, por meio da aplicação do teste U de Mann-Whitney com valor de $p=0,031$, como se observa na tabela 1 . No âmbito geral, é predominante a classificação da remuneração recebida como regular em todos os scores da análise preliminar da Síndrome Burnout, ou seja, a tendência é de que os enfermeiros(as) que atuam nas UTIs deste hospital referente a pesquisa e que possuem a possibilidade de desenvolver a Síndrome de Burnout, bem como estão na fase inicial da Síndrome ou a mesma começou a se instalar, classificam a remuneração recebida como regular.

\section{Discussão}

Os resultados encontrados nesta pesquisa, em conjunto com pontuados na literatura entra em consonância com a afirmação pela Health Education Authority, de que a enfermagem é quarta profissão mais estressante do setor púbico, pelo contato direto com o sofrimento do próximo.

Concomitante a essa afirmação, há a contextualização do ambiente da UTI, com tecnologias, inovações para o cuidar, alta demanda de cuidados complexos associados a carga de agentes estressores, como cansaço, insatisfação, a desvalorização social e monetária dos profissionais, falta de recursos humanos e materiais, na qual acarretam sobrecarga de trabalho e diminuição do tempo de descanso, acarretando o estresse ocupacional e tardar exaustão física e emocional (Perniciotti et al., 2020; Sampaioet al., 2021).

Foram pontuados como fatores ambientais inerentes a UTI como preditores da SB a presença constante de ruídos excessivos, a sobrecarga quantitativa de trabalho sobrecarga qualitativa de trabalho referente à alta morbidade e mortalidade dos pacientes e tomadas de decisões que envolvem dilema bioéticos, sendo estes dois últimos itens descritos, considerados como um dos principais fatores de risco para os enfermeiros (Perniciotti et al., 2020).

Apesar de não haver significância do nível de estresse quando comparado aos dois grupos neste estudo, há na literatura, a constatação da significância do nível de estresse elevado para os enfermeiros que trabalham nas enfermarias COVID-19 quando comparado com os que atuam na enfermaria regular, segundo consta o estudo realizado em um hospital universitário de Augsburg na Alemanha, com 75 enfermeiros atuando em enfermarias regulares (30) e em enfermarias COVID-19 (Zerbini et al., 2020). 
Resultados semelhantes a esta pesquisa, quanto a predominância da classificação de stress laboral global, foram evidenciados em um estudo, com 2310 enfermeiros inscritos em atividade na Ordem dos Enfermeiros de Portugal, na qual predominou a classificação de stress laboral global em nível moderado (51\%) seguido de bastante (29.8\%) e elevado (6.4\%), além de pontuarem resultados interessantes quanto as principais fontes de stress por dimensão sendo carreira e remuneração, seguido de lidar com clientes e sobrecarga de trabalho (Lopes, 2017). O que ao observar, em relação ao contexto temporal e situacional deste estudo, podemos visualizar que os três principais focos apontados são os mesmos focos desta pesquisa alterando a ordem de disposição deles, sendo a ordem excesso de trabalho, lidar com clientes, carreira e remuneração.

Ao ressaltar o excesso de trabalho em primeira ordem nesta pesquisa, observa-se a ótica de que a enfermagem é uma das profissões que excedem sua carga de trabalho, decorrente a realização de várias funções exercidas pelos enfermeiros que além da assistência ao paciente, são responsáveis por treinar e capacitar sua equipe, realizar o gerenciamento de insumos e materiais, orientação dos pacientes e familiares, e essa sobrecarga atrelada situação da pandemia do COVID-19, com as mudanças drásticas nas relações sociais e de trabalho, tem contribuído para o desequilíbrio emocional fazendo com que os profissionais de enfermagem sejam mais suscetíveis ao sofrimento psíquico (Nunes, 2020; Pereira, 2021).

$\mathrm{O}$ resultado de prevalência deste estudo ( $82 \%$ dos enfermeiros com a síndrome de burnout em curso) foi semelhante ao de um estudo transversal, descritivo e exploratório, com abordagem quantitativa, realizado na UTI adulto de um Hospital público em Pernambuco, contando com uma amostra por conveniência de 21 enfermeiros, antes do contexto da pandemia, sendo aplicado o questionário JBEILI para análise da incidência de Burnout nestes profissionais, foi constatado uma prevalência da síndrome em $62 \%$ da amostra, os outros $38 \%$ da amostra apresentaram possibilidade para desenvolver a síndrome (Neves et al., 2020).

Em outro estudo realizado em um município da Parnaíba, Piauí, com a aplicação do questionário de Jbeili para avaliar a predisposição da Síndrome de Burnout em profissionais de enfermagem atuantes na UTI, evidenciou que 64\% da amostra tem predisposição para desenvolver a síndrome, $29 \%$ estão na fase inicial e $7 \%$ em uma fase mais avançada, sendo a amostra com $43 \%$ em idade de 31 a 40 anos, $71 \%$ casados, $79 \%$ são do sexo feminino, 51\% têm uma carga horária semanal de 36 horas (Marinho, 2015).

Os resultados pontuados, demonstram a relevância a respeito da prevalência da Síndrome de Burnout, ao longo do tempo, em relação aos enfermeiros (as) intensivistas e observando o resultado nesta pesquisa, quanto a profissionais com predisposição a desenvolver a síndrome, que estão em estágio inicial e fase avançada da síndrome, há distribuições diferentes, contextos diferentes, porém ambos ressaltam a importância sobre o tema, evidenciando a vulnerabilidade do enfermeiro em desenvolver bem como estar em curso da SB, correlacionado ao ambiente e condições laboral da UTI (Marinho, 2015; Vasconcelos \& Martino, 2017).

Apesar de não haver correlação significativa, neste estudo, relacionada às variáveis sociodemográficas, a literatura aponta, que a predominância do sexo feminino em relação a amostra está relacionada ao processo histórico da profissão atrelada com a feminização do cuidado, compondo 85,1 \% do perfil de enfermagem no Brasil (sendo crescente o ingresso de homens na área, na qual respondem por 14,4\%). Constatado também, que as mulheres possuem uma predisposição maior em apresentar a Síndrome de Burnout sendo levado em consideração as alterações fisiológicas inerentes ao sexo feminino como hormônios, bem como o fato de comumente se envolverem mais com os problemas das pessoas para quem prestam o cuidado, concomitante ao fato da conciliação da jornada de trabalho com as tarefas domésticas, na qual acarretam em maior comprometimento em relação ao sono, lazer e descanso, estando mais expostas à fadiga crônica (Oliveira et al., 2017a; Machado et al., 2015; Neves et al., 2020; Oliveira et al., 2017b; Vasconcelos \& Martino, 2017).

Em relação ao estado civil, a literatura aponta que o indivíduo solteiro apresenta maior probabilidade em desenvolver a Síndrome de Burnout, comparado com os que são casados, pois apesar de vivenciarem as mesmas condições de estresse, 
possuem um fator protetor correlacionado ao convívio familiar, sendo uma rede de apoio importante para o enfrentamento de estressores, no entanto é um fator de dualidade, pois os solteiros têm menor incidência de exaustão emocional do que os casados, pelo fato de terem que conciliar o trabalho com a vida familiar (Oliveira et al., 2017).

Quanto a idade, tempo de formação e carga horária diária, há tendencias dentro deste estudo, sendo a correlação do maior score de Burnout com maior idade, maior tempo de graduação, maior tempo na instituição e menor carga horária de trabalho (escala diária de 6 h) estando em consonância com um estudo realizado com 91 enfermeiros de terapia intensiva, evidenciaram maior acometimento de Burnout relacionado a enfermeiros com carga horária de 30 horas semanais, o que difere também com outros achados na literatura nacional, pois os enfermeiros com carga horária elevada, sofrem uma sobrecarga laboral, sendo este um dos fatores que predispõem o desenvolvimento da burnout (Vasconcelos, \& Martino, 2017).

A literatura aponta que os níveis mais altos de Burnout estão relacionados aos profissionais mais jovens e recémformados, atrelados a inexperiência, déficit de autoconfiança e conhecimento e dificuldades de tomada de decisões (Oliveira et al., 2017b; Silva et al., 2020).

Por fim, observa-se por meio de estudos que os fatores sociodemográficos, não são por si só a causa eficiente da Burnout, as mesmas estão atreladas a fatores que interferem na atividade laboral, como a estrutura do local de trabalho, a disponibilidade de recursos humanos e materiais necessários para o atendimento adequado, bem como a autonomia profissional, que somados a estressores, como sobrecarga de trabalho, baixos salários, duplas e longas jornadas, a manutenção de funcionários em setores de baixa afinidade, contextualizando a pandemia -funcionários remanejados desempenhando atividades que não desempenhavam anteriormente, o medo de contaminação, medidas estritas de segurança associadas ao aumento na necessidade de concentração e vigilância se tornam preditores do adoecimento mental e consequentemente a Síndrome de Burnout (Barbosa et al., 2020; Oliveira et al., 2017b).

As principais consequências para os profissionais são graves, em que níveis moderados a altos da SB estão associados a: Distúrbios individuais, como transtorno de estresse pós-traumático (TEPT), abuso de álcool, queixas psicossomáticas, uso de drogas, depressão e ideação suicida; Mudanças comportamentais voltadas à insatisfação no trabalho, falta de comprometimento organizacional e intenção de abandonar o trabalho, o que gera o aumento da rotatividade de profissionais nos hospitais, levando a altos custos organizacionais para a substituição dos funcionários e; Problemas no trabalho, como absenteísmo, piores resultados nas medidas de segurança ao paciente e erros na prática profissional, o que leva à perda de confiança, dificuldades para dormir, redução da satisfação no trabalho, aumento dos níveis de estresse ocupacional e danos à imagem profissional. (Perniciotti et al., 2020).

Os números são alarmantes, perante a situação ocasionada pela pandemia e os agravos psíquicos evidenciados pela OMS em profissionais da área da saúde, a Associação de Medicina Intensiva Brasileira (AMIB), em conjunto com outras sociedades e com o suporte da Federação Mundial de Cuidados Críticos e Intensivos (WFICC) realizou um levantamento sobre ponto de vista dos profissionais que estão atuando na linha de frente no Brasil, realizando um comparativo entre dois períodos: junho de 2020 e março de 2021. O estudo pontuou que durante o pico da primeira onda da Pandemia (COVID-19) 85\% da amostra, apontava exaustão física e emocional, já em 2021, 90\% afirma ver colegas cansados física e emocionalmente, demonstrando que esta condição é frequente e continua sendo (Associação de Medicina Intensiva Brasileira, 2021; Humerez et al., 2020).

Nesta linha, pontua-se a famigerada incógnita "Quem cuidará de quem cuida?", como aponta Souza e ressalta a importância da discussão política a respeito de melhores condições de trabalho para a enfermagem, como a regulamentação da jornada de trabalho e o piso salarial (Souza e Souza, \& Souza, 2020).

Estudos apontam que as instituições devem favorecer intervenções específicas e de maneira precoce ao se identificar qualquer alteração comportamental do profissional e pontuam um conjunto de estratégias importantes, adotadas no 
enfrentamento das condições no período da pandemia, na qual minimizam o estresse ocupacional, sendo essas, a estratégia de Coping (estratégia de enfrentamento, consciente, cognitivo/comportamental, utilizando recursos internos e externos para lidar com situações estressantes) a "sala de bem-estar" ( ações de auto cuidado, apoio social, técnicas de relaxamento físico e mental), o treinamento de habilidades de comunicação, inteligência emocional, gestão participativa, corresponsabilização dos sujeitos, reflexão das práticas de risco, tomada de consciência, bem como outros incentivos, como, diminuição da carga de trabalho, atuação de mais profissionais na equipe para equilibrar a falta de funcionários e aumentos salariais sendo fortes aliados que promovem no profissional satisfação e motivação para entregar o seu melhor para o serviço, na qual exerce (Barros et al.,2021; Miranda, \& Afonso, 2021).

\section{Limitações do estudo}

Pontua-se como fatores limitantes do estudo, a utilização de poucos textos para análise correlacional com a literatura, pela razão da contemporaneidade do tema e a existência de poucos artigos que enfoquem a temática saúde mental, estresse em profissionais de enfermagem que atuam nas unidades de terapia intensiva durante o contexto da pandemia.

Em relação a coleta de dados, houve dificuldade quanto a demora na obtenção das respostas dos formulários pelos enfermeiros, devido a forma de comunicação se dar de forma digital, enviado por e-mail, comunicação escolhida para este estudo em questão precisado no contexto da pandemia.

\section{Conclusão}

Os resultados encontrados neste estudo, pontuam níveis de estresse global de bastante a elevado estresse, bem como, predomina nos enfermeiros das UTIs COVID-19 e demais UTIs a Síndrome de Burnout em fase inicial e início de instalação da síndrome em $82 \%$ dos enfermeiros sendo, que os outros $18 \%$ apresentam predisposição a desenvolver a síndrome.

Números alarmantes, na qual, corroboram para necessidade de investir em medidas de intervenção e estratégias para controle dos níveis de estresse, apresentado pelos profissionais mediante ao contexto em que estão inseridos, minimizando repercussões negativas na saúde mental a longo prazo.

Conclui-se, diante do exposto, a necessidade de estudos mais aprofundados a respeito da sintomatologia do estresse ocupacional e Síndrome de Burnout nos profissionais que atuam nas UTIs, bem como a compreensão dos efeitos negativos a longo prazo, a nível de qualidade da assistência prestada bem como taxas de absenteísmo, índice de rotatividade no setor, falta de recursos humanos e sobrecarga de trabalho, que se gerou e os possíveis efeitos negativos a se refletir em um contexto de pós-pandemia.

\section{Referências}

AMIB. (2021). AMIB apresenta dados atualizados sobre a COVID-19 no Brasil. https://www.amib.org.br/noticia/nid/amib-apresenta-dados-atualizadossobre-a-covid-19-no-brasil/

Barbosa, D. J., Pereira Gomes, M., Assumpção de Souza, F. B., \& Tosoli Gomes, A. M. (2020). Fatores de estresse nos profissionais de enfermagem no combate à pandemia da COVID-19: Síntese de Evidências. Comunicação Ciências Saúde, 31(1), 31-47. https://doi.org/10.51723/ccs.v31iSuppl\%201.651

Barros, K. C. C., Leal, M. S., Moreira, R. C. R., Mercês, A. C. O. C., Reis, U. O. P., \& Costa, J. S. P. (2021). Estresse ocupacional em ambiente hospitalar no cenário da COVID-19: revisão das estratégias de enfrentamento dos trabalhadores de enfermagem. Enfermagem Brasil, 20 (3), 413-425. https://doi.org/10.33233/eb.v20i3.4233

Campos, M. S., Esteves, T. M. S., Oliveira, C. A. S. C., \& Garcia, J. R. (2018). O Estresse Ocupacional no Enfermeiro na Unidade de Terapia Intensiva. RevistaE-F@tec, 8(1).https://fatecgarca.edu.br/ojs/index.php/efatec/article/view/124/118

Costa, D. M. (2020). Os desafios do profissional de enfermagem mediante a covid-19. Gestão \& Tecnologia, 1(30), 19-21. http://faculdadedelta.edu.br/revistas3/index.php/gt/article/view/54/34

Gomes, A. R., Cruz, J. F., \& Cabanelas, S. (2009). Estresse ocupacional em profissionais de saúde: Um estudo com enfermeiros portugueses. Psicologia: Teoria e Pesquisa, 25(3), 307-318. 
Gomes, A. R., \& Teixeira, P. (2016). Questionário de Stress nos Profissionais de Saúde (QSPS). Universidade Minho Escola de Psicologia Campus de Gualtar, Braga.

Gomes, A. R., \& Teixeira, P. (2016). Estresse, avaliação cognitiva e saúde psicológica: Instrumentos de teste para profissionais de saúde. Stress and Health, 32 (2), 167-172. $10.1002 /$ smi.2583

Humerez, D. C., Ohl, R. I., \& Silva, M. C. N. (2020). Saúde mental dos profissionais de enfermagem do Brasil no contexto da pandemia COVID-19: Ação do Conselho Fedeal de Enfermagem. Cogitare Enfermagem, 25, e74115. http://dx.doi.org/10.5380/ce.v25i0.74115

ICD-11 for mortality and Morbidity Statistics. (2021, 05/2021). Problems associated with employment or unemployment- QD85 Burnout. Retrieved January 20, 2022, from http://id.who.int/icd/entity/129180281.

Jbeili, C. (2011). Questionário Jbeili para identificação preliminar da Burnout. 2011. http://www.manoel.pro.br/avaliacaoburnout.pdf

Lopes, H. M. F. (2017). Avaliação Cognitiva, Stress Ocupacional e Burnout em Profissionais de Saúde: Estudo com Enfermeiros (Dissertação de Metrado).

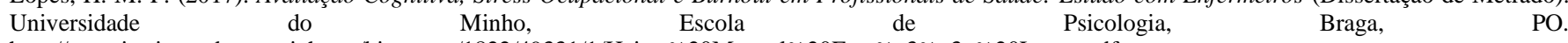
http://repositorium.sdum.uminho.pt/bitstream/1822/49331/1/Heitor\%20Manoel\%20Fraz\%c3\%a3o\%20Lopes.pdf

Luziara, M., \& Jerônimo, C. (2019). Absenteísmo do trabalhador de enfermagem: impactos na saúde do profissional e na assistência. Revista Uniabeu, 12(30), 422-435. https://revista.uniabeu.edu.br/index.php/RU/article/view/3340/pdf

MACHADO, C. S. Estresse e trabalho: aproximações com a psicodinâmica do trabalho. 2020. 113 f. Dissertação (Mestrado em Psicologia) - Universidade Federal de Goiás, Goiânia, 2020.

Machado, M. H., Aguiar Filho, W., Lacerda, W. F., Oliveira, E., Lemos, W., Wermelinger, M., \& Barbosa, C. (2015). Caracteristicas gerais da enfermagem : o perfil sóciodemográfico. Enfermagem em Foco, 7(N. Esp.), 9-14. https://doi.org/10.21675/2357-707X.2016.v7.nESP.686

Marinho, S. A. (2015). Síndrome de Burnout: fatores de risco dos profissionais de enfermagem em uma Uti de um hospital no município de Parnaíba-Pi (Dissertação de Mestrado). Faculdade EST, São Leopoldo. http://tede.est.edu.br/tede/tde_arquivos/1/TDE-2014-10-22T191912Z514/Publico/braga_km_tmp342.pdf

Miranda, A. R. D. O., \& Afonso, M. L. M. (2021). Estresse ocupacional de enfermeiros: uma visão crítica em tempos de pandemia Occupational stress in nurses : a critical view in times of pandemics. Brazilian Journal of Development, 7(4), 34979-35000. https://doi.org/10.34117/bjdv7n4-114

Neves, G. B. C., Santos, O. D. C, Moura, T. A. O., Leoterio, D. S., Martins, P. D. C., Souza, G. T., \& Cruz, E. L. D. (2020). Incidência da síndrome de Burnout em enfermeiros da unidade de terapia intensiva em um hospital público de grande porte em Pernambuco. In S. R. M. Barbosa (Org.). Tecnologia e inovação para o cuidar em enfermagem (pp. 148-155). https://doi.org/10.22533/at.ed.94820261015

Nunes, M. R. (2020). A atuação do enfermeiro em unidade de terapia intensiva na pandemia de COVID-19: relato de experiência. Revista Eletrônica Acervo Saúde, 12(11), e4935. https://doi.org/10.25248/reas.e4935.2020

Oliveira, E. B., Gallasch, C. H., Silva Junior, P. P. A., Oliveira, A. V. R., Valério, R. L., \& Dias, L. B. S. (2017a). Estresse ocupacional e burnout em enfermeiros de um serviço de emergência: A organização do trabalho. Revista Enfermagem UERJ, 25(1), 1-7. https://doi.org/10.12957/reuerj.2017.28842

Oliveira, R. F., Lima, G. G., \& Vilela, G. D. S. (2017b). Incidência da Síndrome de Burnout nos profissionais de enfermagem: uma revisão integrativa. Revista de Enfermagem do Centro-Oeste Mineiro, 7, e1383. https://doi.org/10.19175/recom.v7i0.1383

Pereira, A. (2021). Fatores geradores de estresse ocupacional e seus impactos na saúde dos profissionais de enfermagem que atuam na linha de frente do Covid-19: uma revisão bibliográfica. In P. Silva. Enfermagem: desafios e perspectivas para a integralidade do cuidado (pp. 191-201). https://downloads.editoracientifica.org/books/978-65-89826-82-8.pdf

Pereira, M. D., Torres, E. C., Pereira, M. D., Antunes, P. F. S., \& Costa, C. F. T. (2020). Sofrimento emocional dos Enfermeiros no contexto hospitalar frente à pandemia de COVID-19. Research, Society and Development, 9(8), e67985121. http://dx.doi.org/10.33448/rsd-v9i8.5121

Perniciotti, P., Serrano Júnior, C. V., Guarita, R. V., Morales, R. J., \& Romano, B. W. (2020). Burnout syndrome in healthcare professionals: update on definitions, risk factors and preventive measures. Revista da SBPH, 23(1), 35-52. http://pepsic.bvsalud.org/scielo.php?script=sci_arttext\&pid=S1516$08582020000100005 \& \operatorname{lng}=$ pt\&nrm=iso

Prado, A. D., Peixoto, B. C., Silva, A. M. B., \& Scalia, L. A. M. (2020). A saúde mental dos profissionais de saúde frente à pandemia do COVID-19: uma revisão integrativa. Revista Eletrônica Acervo Saúde, 46 (N. Esp.), e4128. https://doi.org/10.25248/reas.e4128.2020

Sampaio, A. B. O., Cirqueira, A. P., Brito, F. S. L., \& Siqueira, S. M. C. (2021). Burnout Entre Profissionais De Enfermagem Em Época De Coronavírus: O Que Dizem As Evidências Científicas? In S. M. C. Siqueira (Org.), COVID-19: o trabalho dos profissionais da saúde em tempos de pandemia (pp. 64-75). https://downloads.editoracientifica.org/books/978-65-87196-86-2.pdf

Scholze, A., Martins, J., Robazzi, M., Haddad, M., Galdino, M., \& Ribeiro, R. (2017). Estresse ocupacional e fatores associados entre enfermeiros de hospitais públicos. Cogitare Enfermagem, 22(3). http://dx.doi.org/10.5380/ce.v22i3.50238

Silva, C. C., Santos, E. A., Silva, L. A., Silva, L. F., Souza, M. J., \& Sousa, R. S. S. (2020). Perfil dos profissionais de enfermagem portadores da síndrome de Burnout: uma revisão integrativa de literatura. Brazilian Journal of Health Review, 3(3), 4965-4979. https://doi.org/10.34119/bjhrv3n3-079

Souza e Souza, L. P., \& Souza, A. G. (2020). Enfermagem brasileira na linha de frente contra o novo Coronavírus: quem cuidará de quem cuida? Journal of Nursing and Health, 10(4), 20104005. https://doi.org/10.15210/jonah.v10i4.18444

Vasconcelos, E. M., \& Martino, M. M. F. (2017). Predictors of burnout syndrome in intensive care nurses. Revista Gaúcha de Enfermagem, $38(4)$, e65354. https://doi.org/10.1590/1983-1447.2017.04.65354

World Healt Organization. (2018). Nursing Now Campaign. https://www.who.int/hrh/news/2018/NursingNow_launch_press_release.pdf?ua=1

Zerbini, G., Ebigbo, A., Reicherts, P., Kunz, M., \& Messman H. (2020). Psychosocial burden of healthcare professionals in times of COVID-19- survey conducted at the University Hospital Augsburg. German Medical Science, 18, Doc05. 1 https://doi.org/10.3205/000281 